\title{
EUGLENOIDS FROM THE EL FARAFRA OASIS (WESTERN DESERT, EGYPT)
}

\author{
Konrad Wolowski ${ }^{1}$, Abdullah Antar Saber \& Marco Cantonati
}

\begin{abstract}
The paper reports the first detailed study of the diversity of euglenoids recorded from the El Farafra Oasis in the Western Desert of Egypt. Four of the 20 identified species are new records for the Egyptian algal flora: the colorless Peranema inflexum Skuja, and the three pigmented species Euglena adhaerens Matv., Phacus crassus Zakryś \& M. Łukomska and Ph. cristatus Zakryś \& M. Łukomska. A brief description and original documentation are given for each reported morphospecies.
\end{abstract}

Key words: Africa, biodiversity, El-Farafra Oasis, Egypt, Euglenophyta, new records

Konrad Wolowski, Department of Phycology, W. Szafer Institute of Botany, Polish Academy of Sciences, Lubicz 46, 31-512 Kraków, Poland, e-mail: k.wolowski@botany.pl

Abdullah Antar Saber, Botany Department, Faculty of Science, Ain Shams University, Abbassia Square-11566, Cairo, Egypt; e-mail: abdullah_elattar@sci.asu.edu.eg

Marco Cantonati, Museo delle Scienze-MUSE, Limnology and Phycology Section, Corso del Lavoro e della Scienza 3, I-38123 Trento, Italy, and The Academy of Natural Sciences of Drexel University, Patrick Center for Environmental Research, Phycology Section, 1900 Benjamin Franklin Parkway, Philadelphia, PA 19103, U.S.A.; e-mail: marco.cantonati@muse.it

\section{INTRODUCTION}

About a third of the world's land surface has arid or semi-arid climate. Africa is one of the continents with the most dry land. In the northern part of the continent is the largest desert in the world, the Sahara. Oases are a characteristic element of it. Oases are groundwater-sustained islands of verdant fertility in a barren and relatively isolated landscape (Powell et al. 2015; Powell \& Fensham 2016).

On the basis of the $c a 2100$ records in Levanets and Resburg's (2010) bibliography on the freshwater algae of Africa, which includes most of the available literature, we may conclude that the African freshwater algal flora is not sufficiently investigated, particularly in the northeastern region. Wołowski (2012) pointed out that most of the information about the biodiversity of African euglenoids relates mainly to East and Central Africa and narrow strips of Northern and Southern Africa. Recent literature offers a relatively good background on the

\footnotetext{
1 Corresponding author
}

phycological structure of other algal and cyanobacterial groups in African countries (e.g., Touliabah et al. 2002; Shaaban et al. 2013, 2015; Amarouche-Yala et al. 2014; Bere \& Mangadze 2014; Edger et al. 2015; Mansour et al. 2015; Cantonati et al. 2016; Saber \& Cantonati 2016; Saber et al. 2016; Taylor et al. 2016; Janse van Vuuren \& Taylor 2016). Previous studies of euglenoids reported a preponderance of common and cosmopolitan taxa that are considered good bioindicators for eutrophic and polluted inland waters, even those recorded from extreme, isolated or remote habitats. To complete our knowledge of the euglenoids of Africa, however, more in-depth studies are still needed, particularly in oases and other remote habitats. To date only a few papers have been devoted to the euglenoids of Africa (van Oye 1924, 1927; Conrad \& Van Meel 1952; Gerrath \& Denny 1979; Couté \& Iltis 1981; Kadiri 1992, 2004; Kadiri \& Opute 2000; Kemka et al. 2004; Zongo et al. 2006; Nguetsop et al. 2007; Da et al. 2009; Wołowski 2012). Compére's (1975) studies on Lake Chad and its 


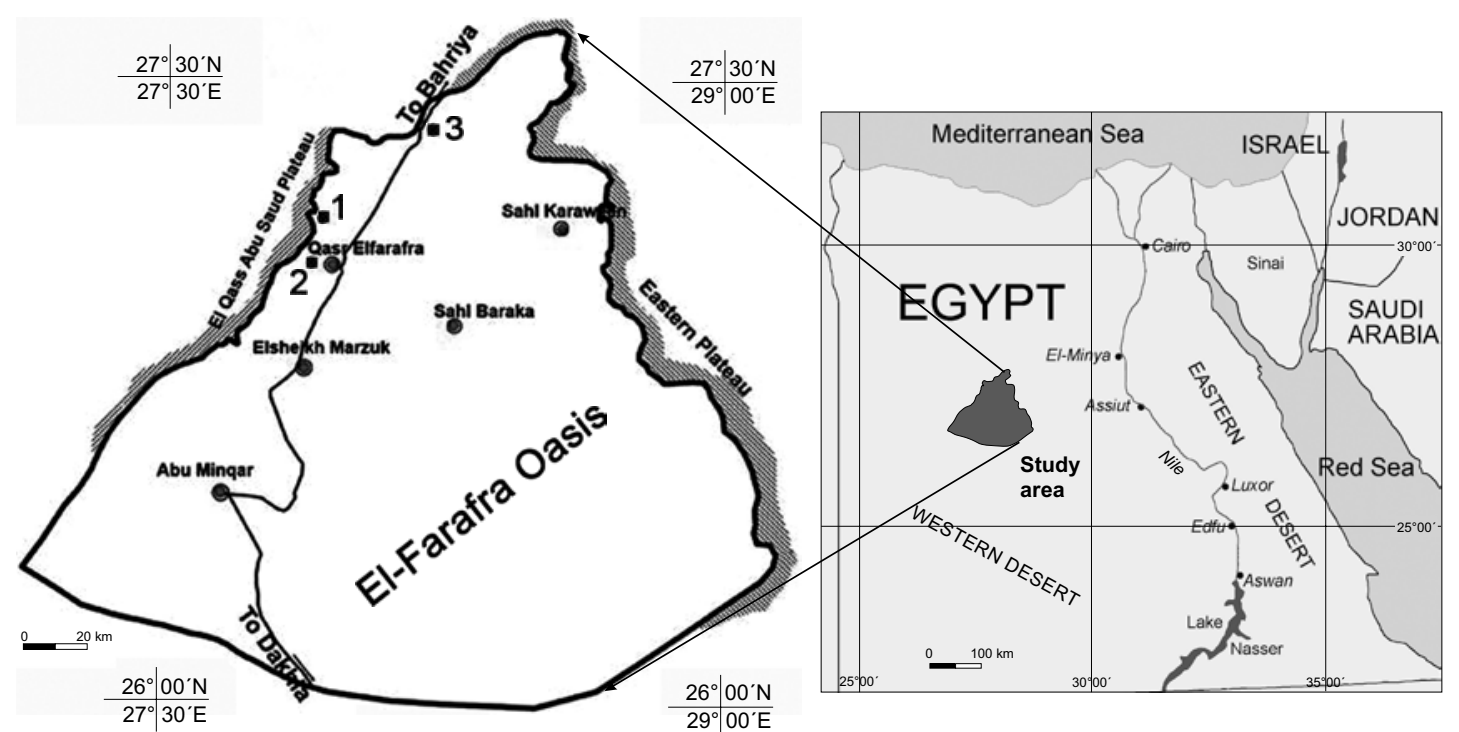

Fig. 1. Location of the studied habitats in El-Farafra Oasis (Western Desert, Egypt). 1 - Abu Nuss Lake, 2 - agricultural drainage at Lewa Soubah village, 3 - Ain Khadra rheocrenic ambient spring in White Desert National Park (WDNP).

surrounding areas included ecological data on 75 different euglenoid species.

There seems to be little information about the species composition and distribution of euglenoids in Egyptian inland waters, particularity in desert oasis habitats. During their phycological investigation of the El-Kharga Oasis, Shaaban and El Habibi (1978) recorded Trachelomonas hispida Skvortsov and $T$. volvocina Ehrenberg from the outlet channels of three drilled wells. A more recent contribution by El-Naghy et al. (2004) on some rice fields in the El-Kharga Oasis documented the presence of only five euglenoids belonging to three genera: Euglena deses var. digrana Zakryś, E. deses var. intermedia G. A. Klebs, E. hemichromata Skuja, Lepocinclis colligera Deflandre and Phacus curvicauda Svirenko. The available information on the algal flora of the Siwa, El-Bahariya and El-Dakhla Oases does not include any species of Euglenophyta (e.g., Shaaban 1985; Shaaban et al. 1997). From the El-Farafra Oasis, Shaaban et al. (2015) reported only Euglena acus (O. F. Müll.) B. Marin \& Melkonian, E. oxyuris (Schmarda) B. Marin \& Melkonian, E. texta Lemmerm. and Lepocinclis fusiformis (H. J. Carter) Lemmerm., from the lakes and drainages investigated during the summer and winter seasons. In general, documentation of euglenoids from the other Egyptian inland water habitats is limited, as confirmed by, for example, a reliable survey of Egyptian freshwaters (Shaaban 1994) which focused mainly on the River Nile and its two branches, pools, lakes and ditches, from which 42 species were reported, 9 of which are considered extinct. Further contributions by El Otify et al. (2003), Gaballa (2014) and El-Kassas and Gharib (2016) also supported this observation. Saber (2010) recorded 14 cosmopolitan species from the main branch of the River Nile and its Rosetta branch. El-Otify (2015) noted the co-occurrence of 5 euglenophytes in his recent study of the algal communities of irrigation and drainage canals in Aswan Province, southern Egypt. Khairy et al. (2015) found 19 euglenoid species in 5 Egyptian Mediterranean lakes.

Here we report our study on the biodiversity of euglenoids inhabiting the Egyptian oases and particularly the El-Farafra Oasis (Fig. 1). We provide detailed information on the morphology, taxonomy and ecology of euglenoids from the hyper-arid desert habitats of the El-Farafra Oasis, including brief descriptions and original documentation for each reported species. 


\section{MATERIALS AND METHODS}

\section{STUDY SITE}

El-Farafra Oasis $\left(26^{\circ} 00^{\prime}-27^{\circ} 30^{\prime} \mathrm{N}, 26^{\circ} 30^{\prime}-29^{\circ} 00^{\prime} \mathrm{E}\right)$, located $c a 650 \mathrm{~km}$ southwest of Cairo, is one of the smallest oases $\left(\mathrm{ca} 10,000 \mathrm{~km}^{2}\right)$ on the limestone plateau occupying the central part of western Egypt (Fig. 1) (El Bastawesy \& Ali 2013). This natural depression is in a hyper-arid region with a hot desert climate; it has $10 \mathrm{~mm}$ or less average annual precipitation and $c a$ $22^{\circ} \mathrm{C}$ mean annual air temperature (MAAT) (Elsheikh 2015; Powell \& Fensham 2016). Groundwater in the El-Farafra Oasis is drawn mainly from natural and artificial wells discharging from the world's largest non-renewable groundwater resource, the Nubian Sandstone Aquifer (Voss \& Soliman 2014). The specimens in this study were collected mainly from three different habitats in the oasis (Figs $1 \& 2$ ): (1) agricultural-waterfed Abu Nuss Lake $\left(27^{\circ} 09^{\prime} 49.4^{\prime \prime} \mathrm{N}, 27^{\circ} 55^{\prime} 46.2^{\prime \prime} \mathrm{E}, 60 \mathrm{~m}\right.$ a.s.1.) in El-Nahda village, (2) an agricultural drainage $\left(27^{\circ} 03^{\prime} 30.5^{\prime \prime} \mathrm{N}, 27^{\circ} 54^{\prime} 03.3^{\prime \prime} \mathrm{E}, 70 \mathrm{~m}\right.$ a.s.1.) in Lewa Soubah village, and (3) a slow-flowing rheocrenic ambient spring, Ain Khadra, also called Ain El-Wadi $\left(27^{\circ} 22^{\prime} 15^{\prime \prime} \mathrm{N}, 28^{\circ} 13^{\prime} 08.8^{\prime \prime} \mathrm{E}, 31 \mathrm{~m}\right.$ a.s.1.) in White Desert National Park (WDNP).

Abu Nuss Lake is one of the main attractions of the El-Farafra Oasis, and is home to abundant birdlife (Fig. 2a). Located in El-Nahda village, it has a total area
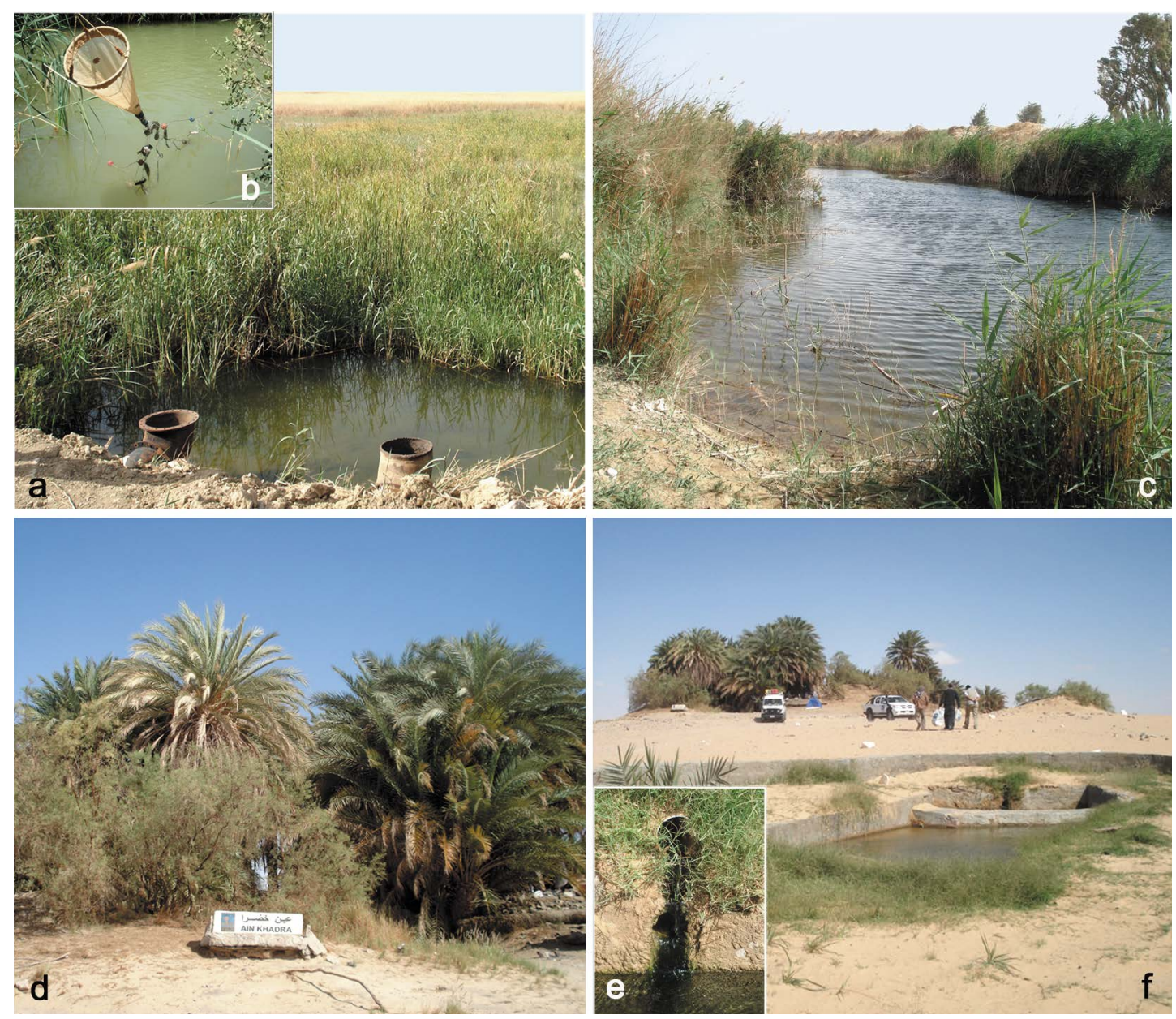

Fig. 2. Landscape views of the sampling sites: a - Abu Nuss Lake with massive growth of the common reed Phragmites australis (Cav.) Trin. ex Steud., b - plankton net hooked by its cone with a fishnet in Abu Nuss Lake, c - agricultural drainage at Lewa Soubah village, $d$ - springhead of Ain Khadra (sustaining vegetated hillocks of palm trees), e - water flowing from Ain Khadra through an underground polypropylene tube, $\mathrm{f}$ - small concrete pool receiving water from Ain Khadra. 
of $c a 15 \mathrm{~km}^{2}$ and ranges in depth between 2 and $3 \mathrm{~m}$. It is $c a 11 \mathrm{~km}$ north of Qasr El-Farafra, the capital and administrative center of El-Farafra Oasis. This lake might be more properly classified as a wetland, and is mainly fed by the water discharged from neighboring agricultural land. It is widely used for fish farming (Fig. 2b). The lake is densely covered with massive growth of the common reed Phragmites australis (Cav.) Trin ex Steud.

The second studied habitat is an agricultural drainage in the vicinity of Lewa Soubah village. The water of this shallow drainage (mean depth $c a 1.5 \mathrm{~m}$ ) is supplied by runoff from agricultural land (Fig. 2c).

The third investigated station, a rheocrenic ambient spring called Ain Khadra, is one of the three main springs that remained active in the WDNP. It is located in the northern extremity of El-Farafra Oasis, ca $35 \mathrm{~km}$ from Qasr El-Farafra. This spring still yields freshwater, with average discharge of $7 \mathrm{~L} \mathrm{~min}^{-1}$ (Powell $\&$ Fensham 2016), and sustains vegetated hillocks rising several meters above the plain (Fig. 2d). The springhead is completely shaded and discharges its water through a large polypropylene tube (Fig. 2e) in a small concrete pool used for recreation and for animals to drink in this isolated desert habitat (Fig. 2f).

\section{HYDROCHEMICAL CHARACTERIZATION}

Water was sampled using polyethylene bottles previously cleaned with ultrapure water and Suprapure nitric acid $(1 \%)$. Water temperature $\left({ }^{\circ} \mathrm{C}\right), \mathrm{pH}$, specific conductivity $\left(\mu \mathrm{S} \mathrm{cm}^{-1}\right)$ and total dissolved solids $\left(\mathrm{mg} \mathrm{l}^{-1}\right)$ were measured in situ using a HANNA HI 991301 calibrated portable Temp/pH/EC/Total Dissolved Solids (TDS) meter. Dissolved oxygen (DO) was determined with a Lutron ${ }^{\circledR}$ YK-22DO in-field calibrated dissolved oxygen meter. Detailed hydrochemical characteristics of the studied habitats, including major ions, nutrients, trace elements and metals were determined by standard procedures and methods (Clescerl et al. 2001). Metals were analyzed with a PerkinElmer Optima 5300 inductively coupled plasma optical emission spectrometer (ICP-OES). $\mathrm{Na}^{+}, \mathrm{K}^{+}, \mathrm{Ca}^{2+}$ and $\mathrm{Mg}^{2+}$ were measured by ionic chromatography (ICS 1500, Dionex Corp.).

Table 1. Main hydrochemical characteristics of the studied habitats in El-Farafra Oasis (Western Desert, Egypt).

\begin{tabular}{|c|c|c|c|}
\hline Parameter & $\begin{array}{c}\text { Abu Nuss Lake } \\
\text { in El-Nahda village }\end{array}$ & $\begin{array}{l}\text { Agricultural drainage } \\
\text { in Lewa Soubah village }\end{array}$ & Ain Khadra \\
\hline Temp. $\left({ }^{\circ} \mathrm{C}\right)$ & 24.5 & 22.4 & 24.0 \\
\hline $\mathrm{pH}$ & 7.3 & 6.6 & 7.25 \\
\hline Conductivity $\left(\mu \mathrm{S} \mathrm{cm}^{-1}\right)$ & 4040 & 5930 & 330 \\
\hline T.D.S. $\left(\mathrm{mg} \mathrm{l}^{-1}\right)$ & 2090 & 2990 & 170 \\
\hline Dissolved oxygen $\left(\mathrm{mg} \mathrm{l}^{-1}\right)$ & 6.2 & 4.4 & 1.5 \\
\hline $\mathrm{Na}^{+}\left(\mathrm{mg} \mathrm{l}^{-1}\right)$ & 502 & 820 & 14.5 \\
\hline $\mathrm{K}^{+}\left(\mathrm{mg} \mathrm{l}^{-1}\right)$ & 49.3 & 62.2 & 20.2 \\
\hline $\mathrm{Ca}^{2+}\left(\mathrm{mg} \mathrm{l}^{-1}\right)$ & 145 & 243 & 10.8 \\
\hline $\mathrm{Mg}^{2+}\left(\mathrm{mg} \mathrm{l}^{-1}\right)$ & 128 & 172 & 14.2 \\
\hline $\mathrm{Cl}^{-}\left(\mathrm{mg} \mathrm{l}^{-1}\right)$ & 944.5 & 2119.32 & 102.03 \\
\hline $\mathrm{SO}_{4}^{2-}\left(\mathrm{mg} \mathrm{l}^{-1}\right)$ & 532.7 & 343.63 & 1.11 \\
\hline $\mathrm{HCO}_{3}^{-}\left(\mathrm{mg} \mathrm{l}^{-1}\right)$ & 163.71 & 100 & 24.37 \\
\hline $\mathrm{CO}_{3}{ }^{2-}\left(\mathrm{mg} \mathrm{l}^{-1}\right)$ & 0.0 & 0.0 & 0.0 \\
\hline $\mathrm{NO}_{2}^{-}\left(\mu \mathrm{g} \mathrm{l}^{-1}\right)$ & 112 & 452 & 0.0 \\
\hline $\mathrm{NO}_{3}^{-}\left(\mu \mathrm{g} \mathrm{l}^{-1}\right)$ & 3760 & 157000 & 400 \\
\hline $\mathrm{NH}_{4}^{+}\left(\mu \mathrm{g} \mathrm{l}^{-1}\right)$ & 60 & 80 & 15 \\
\hline $\mathrm{TP}\left(\mu \mathrm{g} \mathrm{l}^{-1}\right)$ & 325 & 0.0 & 0.0 \\
\hline $\mathrm{SiO}_{2}\left(\mathrm{mg} \mathrm{l}^{-1}\right)$ & 6.8 & 9.2 & 5.5 \\
\hline $\operatorname{Sr}\left(\mu \mathrm{g}^{-1}\right)$ & 3497 & 5860 & 233 \\
\hline $\mathrm{B}\left(\mu \mathrm{g}^{-1}\right)$ & 68.6 & 64.6 & 137 \\
\hline $\operatorname{Li}\left(\mu \mathrm{g}^{-1}\right)$ & 49.1 & 79.6 & 4.0 \\
\hline $\mathrm{Fe}\left(\mu \mathrm{g}^{-1}\right)$ & 32.3 & 24.0 & 29.4 \\
\hline $\operatorname{Mn}\left(\mu \mathrm{g} \mathrm{l}^{-1}\right)$ & 5.1 & 2.9 & 4.6 \\
\hline $\mathrm{Zn}\left(\mu \mathrm{g} \mathrm{l}^{-1}\right)$ & 3.2 & 3.0 & 7.6 \\
\hline $\mathrm{Cu}\left(\mu \mathrm{g} \mathrm{l}^{-1}\right)$ & 0.5 & 0.84 & 0.12 \\
\hline
\end{tabular}


$\mathrm{HCO}_{3}^{-}, \mathrm{CO}_{3}{ }^{2-}, \mathrm{Cl}^{-}$and $\mathrm{SO}_{4}{ }^{2-}$ were determined according to Chapman and Pratt (1978). Nutrients $\left(\mathrm{NO}_{2}^{-}, \mathrm{NO}_{3}^{-}\right.$, $\mathrm{NH}_{4}{ }^{+}, \mathrm{TP}$ ) were measured by molecular absorption spectrometry, and silicates as $\mathrm{SiO}_{2}$ by the molybdosilicate method (Clescerl et al. 2001). The main hydrochemical characteristics of the studied habitats in El-Farafra Oasis are given in Table 1.

\section{COLLECTED MATERIALS}

Euglenoid specimens were sampled April 9-11 2015. The specimens were filtered using a plankton net $(15 \mu \mathrm{m}$ mesh) and transferred in $100 \mathrm{ml}$ sterile clean polyethylene bottles following the method adopted by Bellinger and Sigee (2010). Fresh specimens were transported chilled in a cooler to the laboratory. The materials were studied and identified using a BEL ${ }^{\circledR}$ Photonics biological light microscope, and later preserved with $4 \%(\mathrm{v} / \mathrm{v})$ formaldehyde solution. All descriptions are based on morphotaxonomic observations of living specimens. Voucher specimens were deposited in Phycology Unit No. 341, Botany Department, Faculty of Science, Ain Shams University, Cairo, Egypt. Diagnostic morphometric characters were measured and photographed using Canon Powershot G12 digital camera; 15 measurements of each morphometric character were made. The specimens were identified using the relevant literature: Starmach (1983), Wołowski (1998), Wołowski and Hindák (2005) and Wołowski (2011).

\section{RESULTS AND DISCUSSION}

In the studied habitats of the El-Farafra Oasis we recorded 20 different species and intraspecific taxa of euglenoids belonging to 8 genera: Peranema Dujard. (1), Euglena Ehrenb. (4),
Euglenaformis M. S. Bennett \& Triemer (1), Euglenaria Karnkowska-Ishikawa, E. W. Linton \& Kwiat. (2), Discoplastis Triemer (1), Lepocinclis Perty (4), Phacus Dujard. (6) and Trachelomonas Ehrenb. (1) (Table 2). All these taxa have a cosmopolitan distribution and are known to thrive in moderately to highly polluted waters rich in organic matter (Wołowski 2011; Duangjan et al. 2014; Triemer \& Zakryś 2015). Our results confirm that these euglenoids usually prefer, and proliferate in, warm and stagnant waterbodies (e.g., Wołowski 1998, Ciugulea \& Triemer 2010; Wołowski et al. 2013; Shaaban et al. 2015; Varol \& Şen 2016). These habitat conditions are observed mainly in two of the three studied habitats: Abu Nuss Lake and the agricultural drainage, which showed high concentrations of $\mathrm{N}$ and $\mathrm{P}$ compounds (see Table 1 for more details). Only Trachelomonas volvocina Ehrenb. var. volvocina was found in the rheocrenic ambient spring known as Ain Khadra. Shaaban and El Habibi (1978) documented similar findings in his study of some drilled wells in the El-Kharga Oasis, where only T. hispida (Perty) F. Stein and T. volvocina were recorded. Wołowski (1998, 2011) and Poniewozik (2009) reported that the genus Trachelomonas Ehrenb. mostly prefers colder and clean waters, although it can also be found in warmer and polluted waters.

Phacus crassus Zakryś \& M. Łukomska and Ph. cristatus Zakryś \& M. Łukomska were previously included in the species complex Ph.longicauda in assessments of Egyptian euglenoids

Table 2. Euglenoid taxa reported from various sites of El-Farafra Oasis (I - Abu Nuss Lake, II - agricultural drainage, III - Ain Khadra ambient spring).

\begin{tabular}{|c|c|c|c|c|c|}
\hline \multirow{2}{*}{ Figs } & \multirow{2}{*}{ Taxon } & \multirow{2}{*}{ Taxonomical descriptions } & \multicolumn{3}{|c|}{ Sites and occurrence } \\
\hline & & & I & II & III \\
\hline $3 a, b$ & Peranema inflexum Skuja & $\begin{array}{l}\text { Cell } 32-25 \mu \mathrm{m} \text { long } \times 8-9 \mu \mathrm{m} \text { wide, colorless, various } \\
\text { in shape; common }\end{array}$ & + & - & - \\
\hline $3 c, d$ & Euglena adhaerens Matvienko & $\begin{array}{l}\text { Cell } 80-85 \mu \mathrm{m} \text { long } \times 7.5 \mu \mathrm{m} \text { wide, cylindrical; chloro- } \\
\text { plasts discoid; not common }\end{array}$ & + & + & - \\
\hline $3 e, f$ & E. granulata (G. A. Klebs) F. Schmitz & $\begin{array}{l}\text { Cell } 90-105 \mu \mathrm{m} \text { long } \times 25-30 \mu \mathrm{m} \text { wideW, spindle- } \\
\text { shaped; flexible; chloroplasts disc-shaped with diplopyr- } \\
\text { enoids; common }\end{array}$ & + & - & - \\
\hline $3 g$ & E. cf. geniculata Dujard. & $\begin{array}{l}\text { Cell } 75-80 \mu \mathrm{m} \text { long } \times 17.5 \mu \mathrm{m} \text { wide, cylindrical; two } \\
\text { stellate chloroplasts but slightly damaged; common }\end{array}$ & + & - & - \\
\hline $3 \mathrm{~h}$ & Euglena sp. & Cell $40-45 \mu \mathrm{m}$ long $\times 25-30 \mu \mathrm{m}$ wide & + & + & - \\
\hline
\end{tabular}


Table 2. Continued.

\begin{tabular}{|c|c|c|c|c|c|}
\hline \multirow{2}{*}{ Figs } & \multirow{2}{*}{ Taxon } & \multirow{2}{*}{ Taxonomical descriptions } & \multicolumn{3}{|c|}{ Sites and occurrence } \\
\hline & & & I & II & III \\
\hline $3 \mathrm{i}, \mathrm{j}$ & $\begin{array}{l}\text { Euglenamorfis proxima (P. A. Dang.) } \\
\text { M. S. Bennett \& Triemer }\end{array}$ & $\begin{array}{l}\text { Cell } 52-55 \mu \mathrm{m} \text { long } \times 17.5-22.5 \mu \mathrm{m} \text { wide, spindle- } \\
\text { shaped; chloroplasts discoid, lacking pyrenoids, several } \\
\text { paramylon grains; common }\end{array}$ & + & + & - \\
\hline $3 \mathrm{k}, 1$ & $\begin{array}{l}\text { Euglenaria caudata (E. F. W. } \\
\text { Hübner) Karnkowska-Ishikawa, } \\
\text { E. W. Linton \& Kwiat. }\end{array}$ & $\begin{array}{l}\text { Cell } 100-110 \mu \mathrm{m} \text { long } \times 27.5-30.0 \mu \mathrm{m} \text { wide, spindle- } \\
\text { shaped, chloroplasts discoid with diplopyrenoids; very } \\
\text { common }\end{array}$ & + & - & - \\
\hline $3 m$ & $\begin{array}{l}\text { E. cf. clavata (Skuja) Karnkowska- } \\
\text { Ishikawa \& E. W. Linton }\end{array}$ & $\begin{array}{l}\text { Cell } 35-40 \mu \mathrm{m} \text { long } \times 20.0-22.5 \mu \mathrm{m} \text { wide, club-shaped, } \\
\text { chloroplasts discoid and irregularly lobed; common }\end{array}$ & + & + & - \\
\hline $3 n$ & $\begin{array}{l}\text { Discoplastis spathirhyncha (Skuja) } \\
\text { Triemer }\end{array}$ & $\begin{array}{l}\text { Cell } 65-70 \mu \mathrm{m} \text { long } \times 17.5-20.0 \mu \mathrm{m} \text { wide, variable in } \\
\text { shape, usually from spindle to spinning top; chloroplasts } \\
\text { numerous, discoid; not common }\end{array}$ & + & - & - \\
\hline $3 o, p$ & $\begin{array}{l}\text { Lepocinclis acus (O. F. Müller) } \\
\text { B. Marin \& Melkonian }\end{array}$ & $\begin{array}{l}\text { Cell } 75-150 \mu \mathrm{m} \text { long } \times 7.5-10.0 \mu \mathrm{m} \text { wide, spindle- } \\
\text { shaped, narrow, elongated, rigid; chloroplasts numerous, } \\
\text { discoid without pyrenoids; paramylon bodies numerous, } \\
\text { long, rod-shaped; common }\end{array}$ & + & + & - \\
\hline $4 a$ & L. fusiformis (H. J. Carter) Lemmerm. & $\begin{array}{l}\text { Cell } 27.5-30 \mu \mathrm{m} \text { long } \times 18-20 \mu \mathrm{m} \text { wide, citroform, rigid; } \\
\text { chloroplasts disc-shaped without pyrenoid, one large ring- } \\
\text { like paramylon; common }\end{array}$ & + & - & - \\
\hline $4 b-d$ & $\begin{array}{l}\text { L. oxyuris (Schmarda) B. Marin } \\
\text { \& Melkonian }\end{array}$ & $\begin{array}{l}\text { Cell } 132.5-140.0 \mu \mathrm{m} \text { long } \times 12.5-17.5 \mu \mathrm{m} \text { wide, cylin- } \\
\text { drical with hyaline caudus; chloroplasts small, numerous, } \\
\text { discoid, two large paramylon body; common }\end{array}$ & + & + & - \\
\hline $4 \mathrm{e}, \mathrm{f}$ & L. texta (Dujard.) Lemmerm. & $\begin{array}{l}\text { Cell } 37.5-40.0 \mu \mathrm{m} \text { long } \times 27.5-30.0 \mu \mathrm{m} \text { wide, ovoid, } \\
\text { with small depression at apex; chloroplasts discoid } \\
\text { without pyrenoid; paramylon bodies small, numerous, } \\
\text { oval; common }\end{array}$ & + & + & - \\
\hline $4 \mathrm{~g}$ & $\begin{array}{l}\text { Phacus anomalus F. E. Fritsch } \\
\text { \& M. F. Rich }\end{array}$ & $\begin{array}{l}\text { Cell } 25.0-27.5 \mu \mathrm{m} \text { long } \times 15-20 \mu \mathrm{m} \text { wide, irregular } \\
\text { in shape, usually pear-shaped, with bent caudus; chlo- } \\
\text { roplasts discoid, two paramylon bodies; not common }\end{array}$ & + & - & - \\
\hline $4 \mathrm{~h}$ & $\begin{array}{l}\text { Ph. crassus Zakryś \& M. Łukomska } \\
\text { (delimited from the } P h \text {. longicauda } \\
\text { complex) }\end{array}$ & $\begin{array}{l}\text { Cell ca } 60 \mu \mathrm{m} \text { long } \times 27.5-35.0 \mu \mathrm{m} \text { wide, widely oval, } \\
\text { flattened with long straight cauda, with a short } S \text {-shaped } \\
\text { fold; probably common }\end{array}$ & + & - & - \\
\hline $4 \mathrm{i}, \mathrm{j}$ & $\begin{array}{l}\text { Ph. cristatus Zakryś \& M. Łukomska } \\
\text { (delimited from the } P h . \text { longicauda } \\
\text { complex) }\end{array}$ & $\begin{array}{l}\text { Cell } 50-60 \mu \mathrm{m} \text { long } \times 27.5-35.0 \mu \mathrm{m} \text { wide, widely oval, } \\
\text { semi-flattened, with long fold, spirally twisted with long } \\
\text { sharp caudus, chloroplasts discoid, small; paramylon } \\
\text { body ring-like; common }\end{array}$ & + & - & - \\
\hline $4 \mathrm{k}, 1$ & Ph. curvicauda Svirenko & $\begin{array}{l}\text { Cell } 38.8-40.0 \mu \mathrm{m} \text { long } \times 25-30 \mu \mathrm{m} \text { wide, broadly ovoid, } \\
\text { with longitudinal groove, short bent cauda; chloroplasts } \\
\text { small, discoid, two large disc-like paramylon bodies; } \\
\text { common }\end{array}$ & + & + & - \\
\hline $4 \mathrm{~m}$ & Ph. orbicularis K. Hübner & $\begin{array}{l}\text { Cell } 52.5-75.0 \mu \mathrm{m} \text { long } \times 30-50 \text { wide, broadly ovoid, } \\
\text { with sharp slightly bent cauda; chloroplasts small, dis- } \\
\text { coid; one or two disc-like paramylon bodies; common }\end{array}$ & + & + & - \\
\hline $4 n$ & $\begin{array}{l}\text { Ph. pleuronectes (O. F. Müller) } \\
\text { Nitzsch ex Dujard. }\end{array}$ & $\begin{array}{l}\text { Cell } 75-80 \mu \mathrm{m} \text { long } \times 45-50 \mu \mathrm{m} \text { wide, broadly oval } \\
\text { with short slightly bent caudus, slightly twisted; chloro- } \\
\text { plasts numerous, discoid, usually two disc-like paramylon } \\
\text { bodies; common }\end{array}$ & + & + & - \\
\hline 40 & $\begin{array}{l}\text { Trachelomonas cf. volvocina } \\
\text { (Ehrenb.) Ehrenb. }\end{array}$ & $\begin{array}{l}\text { Lorica } 17.5-20.0 \mu \mathrm{m} \text { in diam., smooth, cell with two } \\
\text { parietal chloroplasts, each with pyrenoids; common }\end{array}$ & - & - & + \\
\hline
\end{tabular}



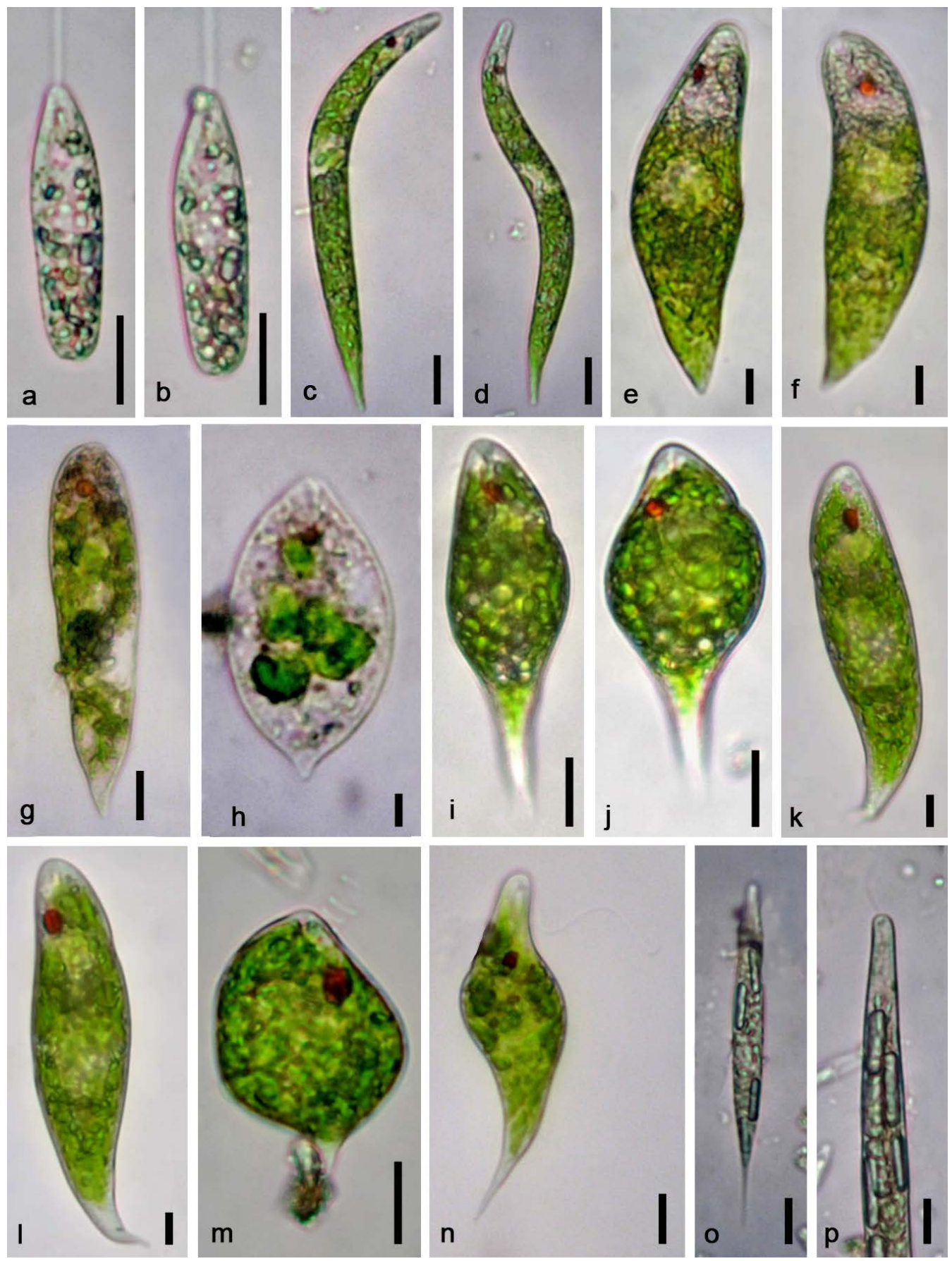

Fig. 3. a \& b-Peranema inflexum Skuja, c \& d-Euglena adhaerens Matvienko, e \& f - E. granulata (G. A. Klebs) F. Schmitz, $\mathrm{g}-$ E. cf. geniculata Dujard., h - Euglena sp., i \& j-Euglenaformis proxima (P. A. Dang.) M. S. Bennett \& Triemer, k \& 1 Euglenaria caudata (E. F. W. Hübner) Karnkowska-Ishikawa, E. W. Linton \& Kwiat., m - E. cf. clavata Karnkowska Ishikawa \& E. W. Linton, $\mathrm{n}$ - Discoplastis spathirhyncha (Skuja) Triemer, o \& p - Lepocinclis acus (O. F. Müller) B. Marin \& Melkonian. Scale bars $=10 \mu \mathrm{m}$. 

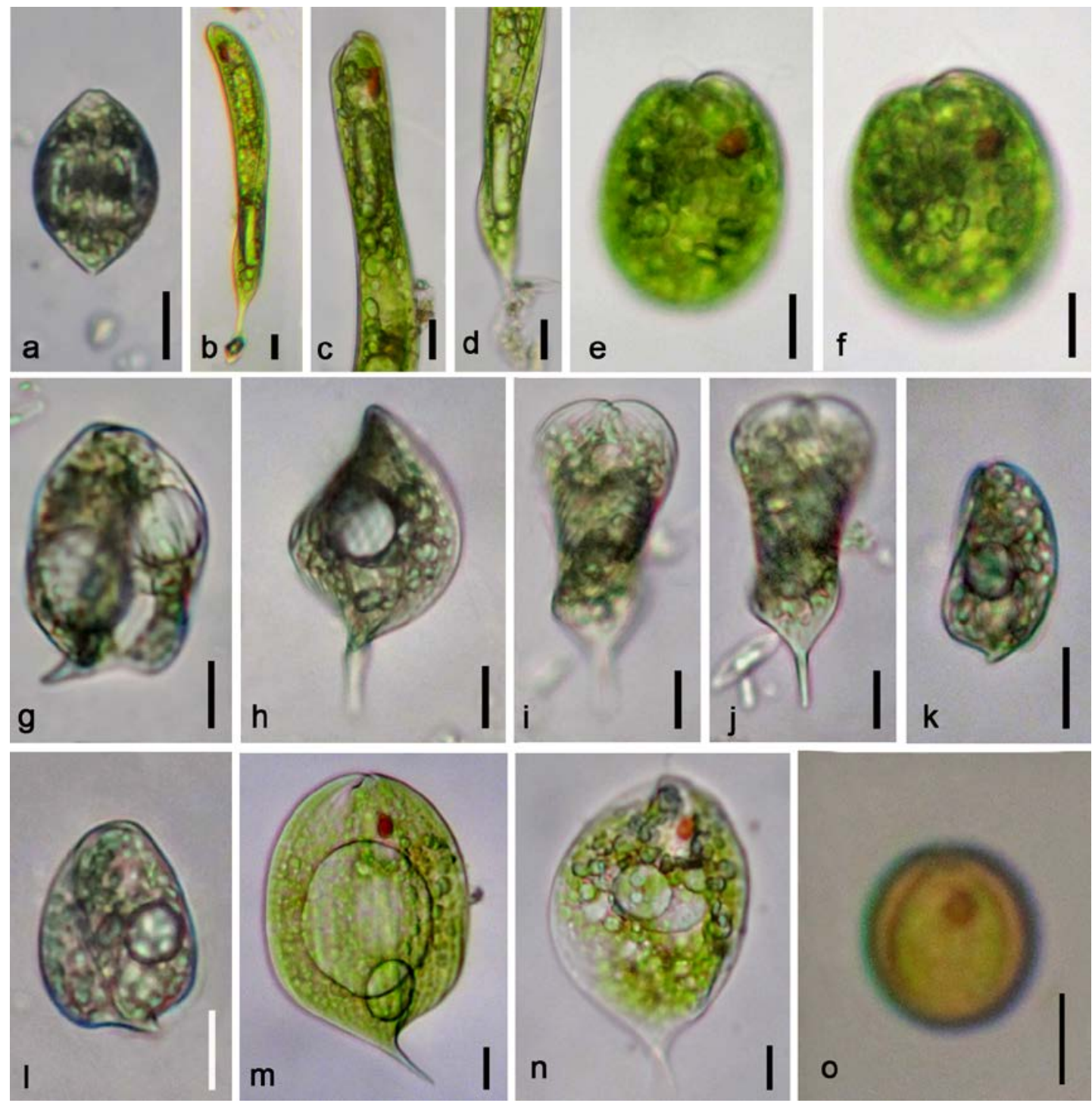

Fig. 4. a - Lepocinclis fusiformis (H. J. Carter) Lemmerm., b-d - L. oxyuris (Schmarda) B. Marin \& Melkonian, e \& f-L. texta (Dujard.) Lemmerm., g - Phacus anomalus F. E. Fritsch \& M. F. Rich, h-Ph. crassus Zakryś \& M. Łukomska, i \& j - Ph. cristatus Zakryś \& M. Łukomska, k \& $1-$ Ph. curvicauda Svirenko, m - Ph. orbicularis K. Hübner, $\mathrm{n}-$ Ph. pleuronectes (O. F. Müller) Nitzsch ex Dujard., o - Trachelomonas cf. volvocina (Ehrenb.) Ehrenb. Scale bars $=10 \mu \mathrm{m}$.

(Saber, unpublished data) but Łukomska-Kowalczyk et al. (2015) recently delimited them as new species based on molecular and morphological. Here we identify them as new records for Egypt.

Detailed observations of the investigated species indicated that their chloroplast shape and structure were somewhat different from those typically observed under laboratory conditions. The chemical properties of the habitats might be responsible for these changes, as suggested by results from Płachno et al. (2015), who found that the shape and arrangement of chloroplasts in some euglenophytes [particularly Euglena viridis (O. F. Müll.) Ehrenb. and E. mutabilis F. Schmitz] 
sampled from heavily polluted streams in Poland varied markedly and did not always fit the original descriptions. Our present work, the first in-depth study of euglenoid species composition in Egypt, points to the need for further investigation of these isolated desert habitats.

\section{Conclusions}

Twenty different euglenoids were identified from samples collected in the El-Farafra Oasis (The Western Desert). Four of these species (Peranema inflexum, Euglena adhaerens, Phacus crassus, Ph. cristatus) are new records for Egypt. All the species found occurred in agricultural and/or humanimpacted habitats. More in-depth studies in Egypt will no doubt produce records of other interesting species, especially in isolated desert habitats.

ACKNOWLEDGEMENTS. We are grateful to the following people and institutions: Dr. Jacopo Gabrieli (Institute for the Dynamics of Environmental Processes of the Italian Research Council, and Department of Environmental Sciences of the University of Venice) for making available the results of complete chemical analyses; the Botany Department, Faculty of Science, Ain Shams University (Egypt), for providing all facilities during the practical work; Mr. Ahmed Kamal, the responsible manager of the WDNP, for his kind support during materials sampling; and Professor Gordana Subakov Simić (Belgrade, Serbia) and the anonymous reviewer for constructive remarks on the manuscript. MC was funded in part by the Autonomous Province of Trento and by the Museo delle Scienze (MUSE), Limnology and Phycology Section, Trento, Italy. Partial financial support was provided by the W. Szafer Institute of Botany, Polish Academy of Sciences.

\section{REFERENCES}

Amarouche-Yala S., Benouadah A., Bentabet A. \& LópezGARCÍA P. 2014. Morphological and phylogenetic diversity of thermophilic cyanobacteria in Algerian hot springs. Extremophiles 18(6): 1035-1047.

Bellinger E. G. \& Sigee D. C. 2010. Freshwater Algae: Identification and Use as Bioindicators. John Wiley \& Sons, Ltd, UK.

Bere T. \& Mangadze T. 2014. Diatom communities in streams draining urban areas: community structure in relation to environmental variables. Trop. Ecol. 55(2): 271-281.
Cantonati M., Saber A. A., Blanco S., El-Gamal D. A., Shehata F. E. \& El-Refaey A. E. A. 2016. Seminavis aegyptiaca sp. nov., a new epilithic diatom species from the estuary of the Damietta Branch of the River Nile (Egypt). In: Pienitz R. \& C. Zimmermann (eds), $24^{\text {th }}$ International Diatom Symposium (program and abstracts), $21^{\text {st }}-26^{\text {th }}$ August 2016, p. 159. Université Laval, Quebec City, Canada.

Chapman H. D. \& Pratt P. F. 1978. Methods of analysis for soil, plants and water. Division of Agricultural Sciences, University of California, Oakland, CA.

Ciugulea I. \& Triemer R. E. 2010. A color atlas of photosynthetic euglenoids. Michigan State University Press, East Lansing.

Clescerl L. S., Greenberg A. E. \& Eaton A. D. (eds) 2001 Standard methods for the examination of water and wastewater, $20^{\text {th }}$ edition. American Public Health Association, Washington, D.C.

CompéRe P. 1975. Algues de la région du lac Tchad. III- Rhodophycées, Euglenophycées, Cryptophycées, Dinophycées, Chrysophycées, Xanthophycées. Cah. O.R.S.T.O.M., ser. Hydrobiol. 9(3): 167-192.

Conrad W. \& Van Meel L. 1952. Matériaux pour une monographie de Trachelomonas Ehrenberg, C., 1834; Strombomonas Deflandre, G., 1930 et Euglena Ehrenberg, C., 1832, genres d'Eugléancées. Mém. Inst. Roy. Sci. Nat. Belgique 124: 1-176.

Couté A. \& ILtis A. 1981. Ultrastructure stéréoscopique de la logette de Trachelomonas récoltés en Côte d'Ivoire. Rev. Hydrobiol. Trop. 14(2): 115-133.

Da K. P., Mascarell G. \& Couté A. 2009. Étude au microscope électronique balayage du genre Trachelomonas (Euglenophyta) dans le Sud-Est de la Côte d'Ivoire. Cryptog. Algol. 30(1): 31-90.

Duanguan K., Wolowski K. \& Peerapornpisal Y. 2014. New records of Phacus and Monomorphina taxa (Euglenophyta) for Thailand. Polish Bot. J. 59(2): 235-247.

Edger R. K., SAleh A. I. \& Edger S. M. 2015. A morphometric diagnosis using continuous characters of Pinnunavis edkuensis, sp. nov. (Bacillariophyta: Bacillariophyceae), a brackish-marine species from Egypt. Phytotaxa 212(1): $1-56$.

El Bastawesy M. \& Ali R. R. 2013. The use of GIS and remote sensing for the assessment of waterlogging in the dryland irrigated catchments of Farafra oasis, Egypt. Hydrological Processes 27: 206-216.

El-Kassas H. \& GHarib S. M. 2016. Studies on spatio-temporal dynamics of phytoplankton in Burullus lagoon, southern Mediterranean coast, Egypt. Egyptian Journal of Experimental Biology (Botany) 12(2): 255-266.

El-Naghy M. A., El-Shahed A. M., Fathi A. A. \& Ahmed G. G. 2004. Algal flora of rice fields at El-Kharga Oasis, Egypt. Egyptian Journal of Phycology 5: 51-69. 
EL-OtiFy A. M. 2015. Water quality assessment of irrigation and drainage systems on the basis of phytoplankton analysis. Catrina 11(1): 9-16.

El-Otify A. M., Shafik H. M. \& SzőKe E. 2003. Analyses of physico-chemical characteristics and phytoplankton communities of Lake Nasser during the last two decades. Acta Bot. Hung. 45(1-2): 75-100.

ElSheIKH A. E. 2015. Mitigation of groundwater level deterioration of the Nubian Sandstone aquifer in Farafra Oasis, Western Desert, Egypt. Environmental Earth Sciences 74(3): 2351-2367.

Gaballa M. M. 2014. Phytoplankton and environmental variables as water quality indicators for El-Temsah Lake, Suez Canal, Egypt. Egyptian Journal of Experimental Biology (Botany) 10(1): 79-85.

Gerrath J. \& Denny P. 1979. Freshwater algae of Sierra Leone. I. Euglenophyta. Nova Hedwigia 31: 525-565.

JANSE VAN VuUREN S. \& TAYlor J. C. 2016. A first record of Aulacoseira ambigua f.japonica (F. Meister) Tuji \& D.M. Williams in South African freshwaters. African J. Aquatic Sci. 41(4): 369-375.

KAdIRI M. O. 1992. Freshwater algae of West Africa. A bibliography, 1956-1991. Polsk. Arch. Hydrobiol. 39: 191-203.

KADIRI M. O. 2004. African freshwater algae a bibliographic up-date. Acta Bot. Hung. 46(1-2): 179-200.

Kadiri M. O. \& Opute F. I. 2000. Euglenoids of the Ikpoba reservoir, Nigeria. Biologia (Bratislava) 55(4): 351-355.

Kemka M., Nuiné T., Zébazé-Togouet S. H., Niyitegeka, Nola M., Monkiedje A., Demanou J. \& Foto MenboHAN S. 2004. Phytoplankton of the Yaounde municipal lake (Cameroon) ecological succession and populations structure. Rev. Sci. Eau 17(3): 301-316.

Khairy H. M., Shaltout K. H., El-Sheekh M. M. \& Eassa D. I. 2015. Algal diversity of the Mediterranean lakes in Egypt. In: International Conference on Advances in Agricultural, Biological \& Environmental Sciences (AABES2015), July 22-23 2015, London (United Kingdom), pp. 127-135. London.

LeVAnets A. \& VAn Rensburg L. 2010. Non-marine algae of Africa. A bibliography (1799-2010). AndCork Publishers, Potchefstroom, Republic of South Africa.

ŁukomsKa-KowalczyK M., KarnKowsKa A., Milanowski R., ŁACH Ł. \& ZAKRYŚ B. 2015. Delimiting species in the Phacus longicauda complex (Euglenida) through morphological and molecular analyses. J. Phycol. 51(6): 1147-1157.

Mansour H. A., Shaaban A. M. \& Saber A. A. 2015. Effect of some environmental factors on the distributions and chlorophyll contents of fresh water phytoplankton of the River Nile before El-Qanater El-Khairia Barrage, Egypt. Egypt. J. Bot. 55(1): 45-60.

Nguetsop V. F., Fonkou T., Nangstong V. M. \& Pinta J. Y. 2007. Relationships between algae taxa and physico- chemical characteristic of water in wetlands and water bodies. Cameroon Journal of Experimental Biology 3(2): $70-79$.

Plachno B. J., Wolowski K., Augustynowicz J. \& ŁukaSZEK M. 2015. Diversity of algae in a thallium and other heavy metals-polluted environment. Ann. Limnol. 51(2): 139-146.

PoniewozIK M. 2009. Taxonomical diversity within Trachelomonas genus in a former, small clay-pit. Fragm. Florist. Geobot. Polonica 16(2): 415-424 (in Polish with English summary).

Powell O. \& Fensham R. 2016. The history and fate of the Nubian Sandstone Aquifer springs in the oasis depressions of the Western Desert, Egypt. Hydrogeology Journal 24(2): 395-406.

Powell O., Silcock J. \& Fensham R. 2015. Oases to oblivion: the rapid demise of springs in the south-eastern Great Artesian Basin, Australia. Groundwater 53(1):171-178.

SABER A. A. 2010. Algal biodiversity of Rosetta branch of River Nile. M.Sc. Thesis, Faculty of Science, Ain Shams University, Egypt.

Saber A. A. \& Cantonati M. 2016. The phycological biodiversity of springs and wells in Egyptian oases, and first attempts to use it for assessment purposes. In: $33^{\text {rd }}$ International Society of Limnology (SIL) Congress, $31^{\text {st }} \mathrm{July-5^{ \text {th } }}$ August 2016, p. 312. Torino, Italy. http://www.sil2016.it/ files/3214/7272/2565/33rd_SIL_Congress_2016_-_Book of_Abstracts.pdf

SABer A.A., Ichihara K. \& CANTonati M. 2016. Molecular phylogeny and detailed morphological analysis of two freshwater Rhizoclonium strains from contrasting spring types in Egypt and Italy. Plant Biosystems (2016): 1-13. DOI: 10.1080/11263504.2016.1211195.

ShaAban A. S. 1985. The algal flora of Egyptian oases. II. On the algae of Siwa oasis. Proceedings of Egyptian Botanical Society 4: 1-10.

ShaAban A. S. 1994. Freshwater algae of Egypt. The United Nations Environmental Programme, National Biodiversity Unit, Biological Diversity of Egypt. GF/6105-92-02-2205.

ShaAban A. S. \& El Habibi A. I978. The algal flora of Egyptian oases. I. The algal flora of Kharga oasis. Bulletin of Desert Institute 28(1): 227-232.

Shaaban A. S., Hamed A. F. \& Fumanti B. 1997. The algal flora of Egyptian Oases. III. The algal flora of the thermal springs of Bahariya oasis. Egyptian Journal of Aquatic Biology and Fisheries 1(1): 85-98.

ShaAban A. S., Mansour H. A. \& SAber A. A. 2015. Unveiling algal biodiversity of El-Farafra Oasis (Western Desert, Egypt) and potential relevance of its use in water bioassessment: special interest on springs and drilled wells. Egyptian Journal of Phycology 16: 47-75.

Shaaban A. S., Cantonati M., Mansour H. A. \& Saber A. A. 2013. Diatoms from different freshwater habitats of El-Farafra Oasis (Egypt), with special attention to wells 
and hot springs. In: F. Rimet, A. Bouchez, L. Ector \& B. Montuelle (eds), 32 crme Colloque de l'Association des Diatomistes de Langue Française \& $7^{\text {th }}$ Central European Diatom Meeting, $16^{\text {th }}-20^{\text {th }}$ September 2013, pp. 207-209. Thonon-les-Bains, France.

Starmach K. 1983. Euglenophyta. Flora Slodkowodna Polski. 3. Państwowe Wydawnictwo Naukowe, Kraków.

TAYlor J. C., CocquYt C. \& Mayama S. 2016. New and interesting Eunotia (Bacillariophya) taxa from the Democratic Republic of the Congo, tropical central Africa. Plant Ecology and Evolution 149(3): 291-307.

Touliabah H., Shafik H. M., Gab-Allah M. M. \& Taylor W. C. 2002. Phytoplankton and some abiotic features of El-Bardawil Lake, Sinai, Egypt. African J. Aquatic Sci. 27(2): 97-105.

Triemer R. E. \& Zakryś B. 2015. Photosynthetic Euglenoids. In: J. WeHr, R. SHEATH \& J. P. KocioleK (eds), Freshwater Algae of North America, $2^{\text {nd }}$ ed., pp. 459-483. Elsevier, Amsterdam etc.

van Oye P. 1924. Note sur l'Euglena acus Ehrenberg. Bull. Soc. Roy. Bot. Belgique 56(2): 124-132.

VAN OYe P. 1927. Le genre Trachelomonas au Congo Belge. Bull. Soc. Roy. Bot. Belgique 59(2): 164-185.

VArol M. \& ŞEN B. 2016. New records of Euglenophyceae for Turkish freshwater algae. Turkish Journal of Fisheries and Aquatic Sciences 16(2): 219-225.

Voss C. I. \& Soliman S. M. 2014. The transboundary nonrenewable Nubian Aquifer System of Chad, Egypt, Libya and Sudan: classical groundwater questions and parsimonious hydrogeologic analysis and modeling. Hydrogeology Journal 22(2): 441-468.

WolowsKi K. 1998. Taxonomic and environmental study on euglenophytes of the Kraków-Częstochowa upland (southern Poland). Fragm. Florist. Geobot. Suppl. 6: 1-192.

Wolowski K. 2011. Phylum Euglenophyta. In: D. M. JoHn, B. A. Whitton \& A. Brook (eds), The freshwater Algal Flora of the British Isles, $2^{\text {nd }}$ ed., pp. 181-239. Cambridge University Press, Cambridge.

Wolowski K. 2012. Euglenophytes from a fishpond in the Republic of Cameroon (West-Central Africa). In: K. WoLOWSKi, I. KaCZMarska, J. M. Ehrman \& A. Z. WoJTAL (eds), Current advances in algal taxonomy and its applications: phylogenetic, ecological and applied perspective, $\mathrm{pp}$. 175-183. W. Szafer Institute of Botany, Polish Academy of Sciences, Kraków.

Wolowski K. \& Hindák F. 2005. Atlas of Euglenophytes. Veda, Bratislava.

Wolowski K., Poniewozik M. \& Walne P. L. 2013. Pigmented euglenophytes of the genera Euglena, Euglenaria, Lepocinclis, Phacus and Monomorphina from the southeastern United States. Polish Bot. J. 58(2): $659-685$.

Zongo F., Mascarell G. \& Couté A. 2006. Strombomonas guinkoi sp. nova (Euglenophyta), une nouvelle euglénophycée d'eau douce du Burkina Faso (Afrique de l'Ouest). Algol. Stud. 119: 17-27. 